\title{
Preface: Orbits around asteroid
}

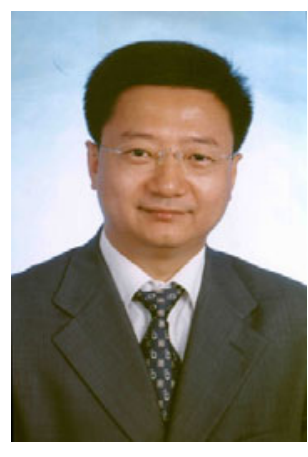

\section{GUEST EDITOR: Junfeng Li, Professor of Astrodynamics}

\section{RESEARCH INTERESTS:}

Global trajectory optimization; Satellite formation flying; Liquid sloshing Stability of Motion

\section{School of Aerospace, Tsinghua University, 100084 Beijing, China} e-mail: lijunf@tsinghua.edu.cn
One of the core issues in modern celestial mechanics is the orbital dynamics in the near-regime gravitational field of asteroids, which provides deep insights into the mathematical nature of a class of nonlinear systems, and plays as a critical basis for in situ explorations of different science goals. Lots of efforts have been made to reveal the characteristics of orbital motion in the vicinity of asteroids, and to improve the skills of asteroid research in methodology. This theme issue of the Acta Mechanica Sinica contains a serie of innovative papers regarding the orbital dynamics around asteroids and the approach to model asteroids from close-up optical images.

H.-X. Baoyin et al. reviewed the latest developments on the dynamics, control and navigation of asteroid reconnaissance orbits, including the heliocentric transfer orbit and near asteroid orbit. The advances in the optimization techniques for transfer segments of asteroid explorations were highlighted and discussed in detail. Several important progresses were then referred. The authors gave comments to the key techniques in future asteroid explorations, and prospected the possible hotspots in next couple of years. W.D. Hu et al. summarized a few cases of orbits for which the averaging method may apply, and presented the averaging conditions for these special cases. In this study, the averaging method was also extended into a few classes of near resonant orbits, and the major conclusions of this analysis showed a strong link to the results of stability regions in the numerical studies, that the near circular 1:2 resonant orbit is always unstable. X.-Y. Li et al. proposed a new method to model the shape of an asteroid from the optical images closeup the asteroid. This method was then applied to obtain the preliminary shape model of Asteroid 4179 Toutatis from the high-resolution images taken by Chang'E-2 during the flyby.
The basic characters were first extracted from the optical images, and then these characters were applied to improve the similarity between the shape model and Toutatis based on the original radar shape. In addition, the correlated spherical harmonic coefficients were derived from the preliminary polyhedron model for further application in the further study of asteroid shape. Y. Yu et al. developed an event-driving model to describe the motion of a small particle/probe governed by the irregular gravitational field and geomorphology close to the surface of a real asteroid. This method was then implemented in numeric and applied to the analysis of surface dynamical environment of Asteroid 1620 Geographos. The results showed a significant connection between the behaviors of surface motion trajectories and the geometry of asteroid surface. The performance of vehicles delivered to the surface of asteroids was concerned in this paper. The results of Monte Carlo simulations indicated the high-risk region on Geographos' surface, and then the descending trajectories of the surface vehicle were investigated to find the safe deploying height. X.-Y. Wang et al. presented a classification for the orbits near an asteroid, serving as an indicator for designing the probe's orbit in the practical missions. The influence of non-spherical perturbations was analyzed based on an approximated form of gravitational field. Numerical simulations presented the orbits around Asteroid 216 Kleopatra, and the results showed consistent with the analysis.

The contributions included in this special issue referred to several important aspects in current asteroid research, which has become a field of high concern and will continue to be a fruitful area in the foreseeable future. It has been the privilege of the guest editors to organize this special issue. We wish to thank all the authors and reviewers for their valuable contributions. 